\title{
MediNet: A Mobile Healthcare Management System for the Caribbean Region
}

\author{
Permanand Mohan and Salys Sultan \\ The University of The West Indies, St. Augustine, Trinidad and Tobago
}

\begin{abstract}
There are numerous challenges confronting the healthcare systems of Caribbean countries. The public healthcare systems in the region currently face a crisis due to factors such as shortages of medical staff, lack of proper facilities, and insufficient funding. Therefore, new approaches to managing healthcare and promoting healthier lifestyle practices are needed to address this problem. This paper will discuss MediNet, a mobile healthcare management system being developed at the St. Augustine Campus of the University of the West Indies to provide an alternative approach to healthcare in the Caribbean. The initial focus of the system is on chronic non-communicable diseases such as diabetes and cardio-vascular disease. The learning and feedback from the first prototype will be used to build a full-fledged system capable of handling other diseases.
\end{abstract}

Index Terms-Chronic Non-communicable Diseases, Healthcare Management System, Mobile Healthcare.

\section{INTRODUCTION}

$\mathrm{T}$ he liberalization of telecommunication markets across the Caribbean has caused the cost of owning a mobile phone to drop significantly, sending market penetration to an unprecedented high. Almost everyone today owns a mobile phone and some even own two. Cellular phone technology plays an important role in MediNet. Each of the two main Caribbean mobile network carriers across the region have implemented wireless communications infrastructure which has wider reach and appeal to the public than terrestrial lines. It is cheaper to leverage this wireless communications infrastructure to provide a range of services since it bypasses many principal physical development phases encountered in the region such as power grids, fibre optic backbones, and roads. It is primarily this low cost and ubiquity which makes cellular phone technology particularly attractive for developing a healthcare solution based on mobile telephony in this region.

Research [1] has shown that better blood pressure control in diabetic patients who have high blood pressure reduces the risk of: death from long-term complications of diabetes by a third, strokes by more than a third, and serious deterioration of vision by more than a third. Thus, patients suffering from diabetes and hypertension can reduce their morbidity and mortality by maintaining tight control of their blood sugar and blood pressure. If the patients in the Caribbean who suffer with diabetes and cardio-vascular disease can somehow engage in better management of these diseases, this is likely to have a positive impact on their ability to live longer, more meaningful lives.

Therefore, a new approach to managing diabetes and cardio-vascular diseases in patients from the Caribbean is needed. This paper presents a solution that is cost-effective so that it can be adopted by the population throughout the region.

Cellular phone technology plays an important role in the Caribbean-wide Healthcare Management System. The long term objective of the MediNet project is the development of a network that would integrate the medical resources of the entire region thereby promoting the sharing of medical expertise and resources.

\section{THE MEDINET ARCHITECTURE}

In MediNet, patient readings from various measuring devices such as a blood glucose meter and a blood pressure meter are transferred automatically to a cellular phone where it is stored in a local database. The cellular phone transfers the readings to a Web server via GPRS where it is stored permanently in a database. Software on the patient's cellular phone recommends a certain course of action to be taken by the patient depending on the readings obtained. In critical situations, the software may notify other agencies to intervene.

By implementing the system across the Caribbean region, a distributed system is obtained where most of the knowledge concerning the diseases can be shared among countries, perhaps using replication to avoid intra-regional networks costs. However, most of the healthcare services that may be available to a patient may not likely to be in the same country, so it is necessary to create country-wide networks (called MediNets) that will connect to the overall Healthcare Management System. The architecture of each regional system is shown in Figure 1. It is focuses primarily on cardiovascular disease and diabetes but will be extended to other medical diseases in later phases. 


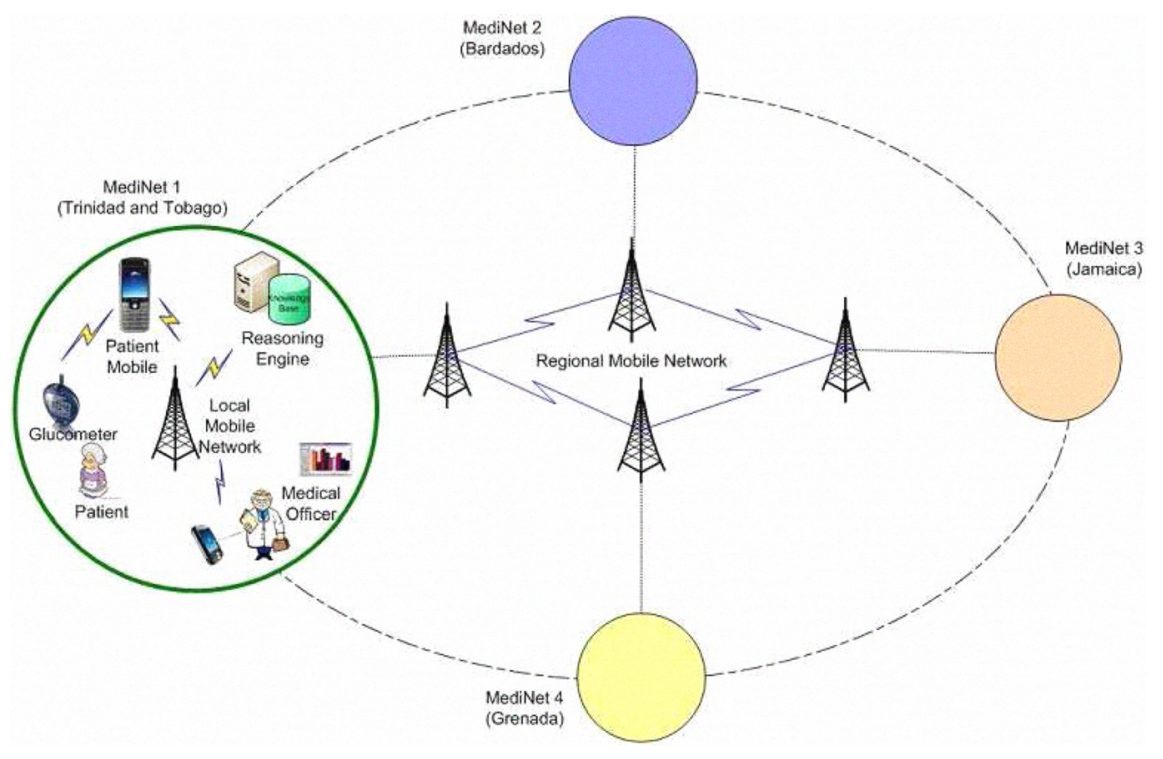

Fig. 1. Regional MediNets Architecture

\section{WORK DONE SOFAR}

The MediNet project is broken up into different stages. The first stage involves a pilot study of the system in one Caribbean country, Trinidad and Tobago. The country comprises two islands, so it provides the opportunity to test the distributive and networking aspects of the system.

For the pilot study, a patient interface called myDR (short for my daily record) [3] was developed for two platforms: Windows Mobile Professional 6.0 running on a HTC Touch smart phone and a Java version that runs on a Nokia 5310 . The Windows Mobile 6 version makes use of many of the features of the smart phone environment, including the stylus based input, graphical user interface and built-in calendar capabilities. The Java version provides the same functionality as the Windows Mobile 6 version; however, it is more textoriented and runs on mobile phones that are more readily available in the Caribbean region. The main objective of the patient interface is to collect the patient's physiological measurements from the respective meters and send the readings to the patient's mobile phone, as well as provide the patient with a personalized recommendation [2] that is generated based on a set of clinical rules.

The next component of the MediNet system is a set of Web services which interact with the patient interface. One service transmits the patient's readings to a database at a Web server. Another is responsible for generating text messages to the patient, patient's health care providers and caregivers. A third is responsible for authenticating users. Other Web services return data for display on the patient interface based on requests made by the patient. A Web service is available that uses a reasoning engine to analyse the history of readings and then generates and sends appropriate feedback based on a set of rules.

The third component of MediNet is an electronic diary to be used by the patient's healthcare providers or the patient herself to remotely monitor the condition of the patient.

Usability trials of the system were conducted in February 2009 , to test the patient interface. The majority of the patients found the system easy to use and said that it was a big improvement on their current monitoring process. Testing of the system with about 15 patients will begin in July 2009 .

\section{FUTURE WORK AND CONCLUSION}

The next stage of the MediNet project involves conducting clinical trials in Trinidad and Tobago with a group of 150-200 patients to prove that the system does impact the life of the patients in a positive way. After these trials, MediNet will then be implemented in a phased basis targeting three other Caribbean countries. The proposed countries are Barbados, Jamaica, and Grenada. Once the networks are deployed and fully tested, clinical trials would then be conducted at a regional level.

The following are some research areas surrounding the deployment of the regional system: security and 'sharing' of the patient data given its distributive nature, the ownership and monitoring of the system since it is a regional solution, the fault tolerance mechanisms in case of network problems and the availability of the system on different mobile platforms throughout the Caribbean.

\section{REFERENCES}

[1] American Diabetes Association. "Implications of the United Kingdom Prospective Diabetes Study" Diabetes Care Jan 02, 2007 vol. 25.

[2] Mohan, P., Marin, D., Sultan, S., \& Deen, A.“ MediNet: Personalizing the Self-Care Process for Patients with Diabetes and Cardiovascular Disease Using Mobile Telephony.” In Proc. 30th Annual International Conference of the IEEE Engineering in Medicine and Biology Society. Vancouver, Canada, 20-24 August 2008, pages 755-758.

[3] Sultan, S., Mohan, P., Sultan, N. 2009. "Managing Change: Experiences from a New e-Health Initiative for Patients with Diabetes and Cardiovascular Disease." In 1st IEEE International WoWMoM Workshop on Interdisciplinary Research on E-Health Services and Systems. Kos, Greece, 15-19 June 2009. 